\title{
Depth-resolved two-dimensional Stokes vectors of backscattered light and Mueller matrices of biological tissue measured with optical coherence tomography
}

\author{
Shuliang Jiao, Gang Yao, and Lihong V. Wang
}

\begin{abstract}
Mueller matrices provide a complete characterization of the optical polarization properties of biological tissue. A polarization-sensitive optical coherence tomography (OCT) system was built and used to investigate the optical polarization properties of biological tissues and other turbid media. The apparent degree of polarization (DOP) of the backscattered light was measured with both liquid and solid scattering samples. The DOP maintains the value of unity within the detectable depth for the solid sample, whereas the DOP decreases with the optical depth for the liquid sample. Two-dimensional depthresolved images of both the Stokes vectors of the backscattered light and the full Mueller matrices of biological tissue were measured with this system. These polarization measurements revealed some tissue structures that are not perceptible with standard OCT. (c) 2000 Optical Society of America OCIS codes: $120.2130,170.4500,260.5430,290.7050$.
\end{abstract}

\section{Introduction}

Optical coherence tomography (OCT) is an important noninvasive medical imaging modality that can reveal the subsurface structures of biological tissues. High spatial resolution $(\sim 1 \mu \mathrm{m})$ and a high scanning speed (at the video rate) were achieved over the past few years. ${ }^{1,2}$ Recently, polarization-sensitive OCT emerged as a very attractive branch of OCT. ${ }^{3-7}$ Polarization-sensitive OCT can provide more information about the optical properties of biological tissues than can standard OCT. Results of these studies reveal the importance of polarization as a contrast mechanism. As is known in polarimetry, Stokes vectors and Mueller matrices ${ }^{8}$ provide complete representations of the polarization properties of light and optical samples, respectively. By measuring the Stokes vectors of the light that is backscattered from biological tissues and calculating the Mueller matrix from the Stokes vectors, one can obtain a complete characterization of the optical po-

The authors are with the Optical Imaging Laboratory, Biomedical Engineering Program, Texas A\&M University, 3120 TAMU, College Station, Texas 77843-3120. L. V. Wang's e-mail address is lwang@tamu.edu.

Received 4 April 2000; revised manuscript received 11 August 2000.

0003-6935/00/346318-07\$15.00/0

(C) 2000 Optical Society of America larization properties of the tissue. With the combination of Mueller matrix measurements and OCT, one can obtain the Mueller matrix of a sample with OCT resolution. Yao and Wang ${ }^{7}$ first reported the measurement of two-dimensional (2-D) depthresolved Mueller matrices of biological tissue with OCT. The results revealed some structures that were not observable with standard OCT. Therefore Mueller matrices provide a unique approach to characterizing biological tissue.

In this paper, we give a detailed account of our novel, to our knowledge, polarization-sensitive OCT system for characterizing the polarization properties with both depth and lateral resolution of biological tissues and other turbid media. A concise relation between the Stokes vectors and the Mueller matrix in our measurement approach was derived. We measured the Stokes vectors of light backscattered from solid biological tissue and liquid turbid media. The apparent degree of polarization (DOP) was calculated from the Stokes vectors. The calculated DOP results revealed the differences between solid and liquid in the DOP as a function of the optical depth. An explanation for the mechanism contributing to this difference is postulated. We also measured 2-D images of both the Stokes vectors for different incident polarization states and the full $4 \times 4$ Mueller matrix of a piece of bone from the head of a croaker fish. 


\section{Stokes Vectors and Mueller Matrices}

In polarimetry the Stokes vector $\mathbf{S}$ of a light beam is constructed on the basis of six flux measurements with different polarization analyzers in front of the detector by

$$
\mathbf{S}=\left(\begin{array}{c}
I_{H}+I_{V} \\
I_{H}-I_{V} \\
I_{P}-I_{M} \\
I_{R}-I_{L}
\end{array}\right)
$$

where $I_{H}, I_{V}, I_{P}, I_{M}, I_{R}$, and $I_{L}$ are the light intensities measured with a horizontal linear polarizer, a vertical linear polarizer, a $+45^{\circ}$ linear polarizer, a $-45^{\circ}$ linear polarizer, a right-circular analyzer, and a leftcircular analyzer, respectively, in front of the detector. Because of the relations $I_{H}+I_{V}=I_{P}+I_{M}=$ $I_{R}+I_{L}=I$, where $I$ is the intensity of the light beam measured without any analyzer in front of the detector, a Stokes vector can be determined by four independent measurements, for example, $I_{H}, I_{V}, I_{P}$, and $I_{R}$ :

$$
\mathbf{S}=\left(\begin{array}{c}
S_{0} \\
S_{1} \\
S_{2} \\
S_{3}
\end{array}\right)=\left(\begin{array}{c}
I_{H}+I_{V} \\
I_{H}-I_{V} \\
2 I_{P}-\left(I_{H}+I_{V}\right) \\
2 I_{R}-\left(I_{H}+I_{V}\right)
\end{array}\right)
$$

From the Stokes vector, we derive the DOP, the degree of linear polarization (DOLP), and the degree of circular polarization (DOCP) as

$$
\begin{aligned}
\mathrm{DOP} & =\frac{\left(S_{1}{ }^{2}+{S_{2}}^{2}+S_{3}{ }^{2}\right)^{1 / 2}}{S_{0}}, \\
\mathrm{DOLP} & =\frac{\left(S_{1}{ }^{2}+S_{2}{ }^{2}\right)^{1 / 2}}{S_{0}}, \\
\mathrm{DOCP} & =\frac{S_{3}}{S_{0}} .
\end{aligned}
$$

The Mueller matrix $\mathbf{M}$ of a sample transforms an incident Stokes vector into the corresponding output Stokes vector

$$
\mathbf{S}_{\text {out }}=\mathbf{M S}_{\mathrm{in}},
$$

where $\mathbf{S}_{\text {in }}$ and $\mathbf{S}_{\text {out }}$ are the incident and the output Stokes vectors respectively, of the light beam. Obviously, the output Stokes vector varies with the polarization state of the incident beam, but the Mueller matrix is determined by only the sample and the optical path. Conversely, the Mueller matrix can fully characterize the optical polarization properties of the sample. The Mueller matrix can be obtained experimentally by measurements with different combinations of source polarizers and detection analyzers. Because a $4 \times 4$ Mueller matrix has 16 independent elements, at least 16 independent measurements must be acquired to determine a full Mueller matrix.
The Stokes vectors for the four incident polarization states, $H, V, P$, and $R$, are, respectively,

$$
\mathbf{S}_{H i}=\left(\begin{array}{c}
1 \\
1 \\
0 \\
0
\end{array}\right), \quad \mathbf{S}_{V i}=\left(\begin{array}{c}
1 \\
-1 \\
0 \\
0
\end{array}\right), \quad \mathbf{S}_{P i}=\left(\begin{array}{l}
1 \\
0 \\
1 \\
0
\end{array}\right), \quad \mathbf{S}_{R i}=\left(\begin{array}{l}
1 \\
0 \\
0 \\
1
\end{array}\right),
$$

where $H, V, P$, and $R$ represent horizontal linear polarization, vertical linear polarization, $+45^{\circ}$ linear polarization, and right-circular polarization, respectively. We can express the $4 \times 4$ Mueller matrix as $\mathbf{M}=\left[\begin{array}{llll}\mathbf{M}_{0} & \mathbf{M}_{1} & \mathbf{M}_{2} & \mathbf{M}_{3}\end{array}\right]$, where $\mathbf{M}_{0}, \mathbf{M}_{1}, \mathbf{M}_{2}$, and $\mathbf{M}_{3}$ are four column vectors of four elements each. The four output Stokes vectors corresponding to the four incident polarization states $H, V, P$, and $R$ are denoted respectively by $\mathbf{S}_{H}, \mathbf{S}_{V}, \mathbf{S}_{P}$, and $\mathbf{S}_{R}$. These four output Stokes vectors are experimentally measured based on Eq. (2) and can be expressed as

$$
\begin{aligned}
\mathbf{S}_{H} & =\mathbf{M} \mathbf{S}_{H i}=\mathbf{M}_{0}+\mathbf{M}_{1}, \\
\mathbf{S}_{V} & =\mathbf{M} \mathbf{S}_{V i}=\mathbf{M}_{0}-\mathbf{M}_{1}, \\
\mathbf{S}_{P} & =\mathbf{M} \mathbf{S}_{P i}=\mathbf{M}_{0}+\mathbf{M}_{2}, \\
\mathbf{S}_{R} & =\mathbf{M} \mathbf{S}_{R i}=\mathbf{M}_{0}+\mathbf{M}_{3} .
\end{aligned}
$$

The Mueller matrix can then be calculated from the output Stokes vectors:

$$
\begin{aligned}
\mathbf{M}= & \frac{1}{2}\left[\mathbf{S}_{H}+\mathbf{S}_{V}, \mathbf{S}_{H}-\mathbf{S}_{V}, 2 \mathbf{S}_{P}-\left(\mathbf{S}_{H}+\mathbf{S}_{V}\right),\right. \\
& \left.2 \mathbf{S}_{R}-\left(\mathbf{S}_{H}+\mathbf{S}_{V}\right)\right] .
\end{aligned}
$$

In other words, at least four independent Stokes vectors for different source polarization states must be measured to determine a full Mueller matrix, and each Stokes vector requires four independent intensity measurements with different analyzers.

In an OCT system the interference signal generated by the interaction of the light beams from the reference arm and the sample arm is

$$
\begin{aligned}
I_{\mathrm{OCT}} & =2 \operatorname{Re}\left[\left\langle\mathbf{E}_{s}\left(l_{s}\right) \cdot \mathbf{E}_{r, A} *\left(l_{r}\right)\right\rangle\right] \\
& =2\left[I_{s, A}\left(l_{s}\right) I_{r, A}\right]^{1 / 2}|V(\Delta l)| \cos \left(k_{0} \Delta l\right),
\end{aligned}
$$

where $\mathbf{E}_{s}$ denotes the sample electric field, $\mathbf{E}_{r, A}$ denotes the reference electric field with a polarization state $A, l_{s}$ and $l_{r}$ are the optical path lengths of the sample arm and the reference arm, respectively, $I_{s, A}$ denotes the light intensity of the sample arm that is projected onto the polarization state $A, I_{r, A}$ denotes the light intensity of the reference arm with the polarization state $A, V$ is the temporal coherence function of the field, $\Delta l$ represents the path-length difference between the sample and the reference arms, and $k_{0}$ is the magnitude of the average wave vector. The path-length difference can be converted to approximately the optical depth, which is the physical depth multiplied by the index of refraction, in the sample in a single-scattering-dominated regime. 


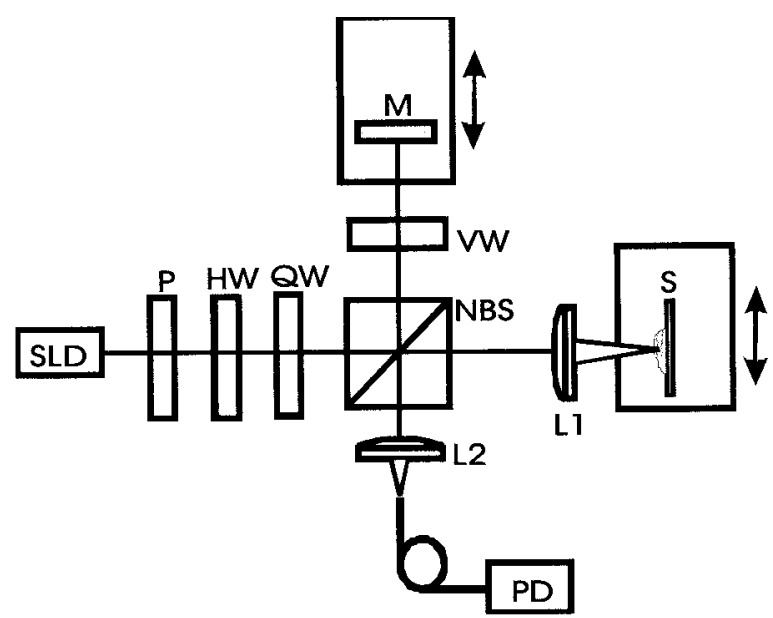

Fig. 1. Schematic of the polarization-sensitive OCT system: SLD, superluminescent diode; P, polarizer; HW, zero-order halfwave plate; $\mathrm{QW}$, zero-order quarter-wave plate; NBS, nonpolarization beam splitter; VW, variable-wave plate; M, mirror; L1 and L2, lenses; PD, photodiode.

For light reflected from a given optical depth in the sample, the following quantity is used to substitute for the time-resolved intensity in conventional polarimetry that would otherwise be measured directly by a noninterference analyzer of the polarization state $A$ :

$$
I_{s, A} \propto I_{\mathrm{OCT}}^{2} / I_{r, A} .
$$

\section{Experimental Setup}

A schematic of the OCT system for our studies is shown in Fig. 1. A superluminescent diode laser with a center wavelength of $850 \mathrm{~nm}$ and a FWHM bandwidth of $26 \mathrm{~nm}$ is used as the light source. The light intensity after the polarizer is $400 \mu \mathrm{W}$. After passing through the polarizer, the half-wave plate, and the quarter-wave plate, the light is split by a nonpolarization beam splitter. The sample beam is focused into the sample by objective lens L1 with a numerical aperture of 0.15 . The reference beam passes through a variable-wave plate and is reflected back. The reflected beams from the reference and the sample arms are coupled into a single-mode fiber and detected by a silicon photodiode. The minimum detectable signal of the system is $-100 \mathrm{~dB}$. A single depth scan through $1.5 \mathrm{~mm}$ takes $6 \mathrm{~s}$ in the current system. The depth resolution of this system is 10 $\mu \mathrm{m}$. The step size of the lateral scan is also $10 \mu \mathrm{m}$. The focal-spot size of objective lens L1 is $6.9 \mu \mathrm{m}$ in air, and it is larger in tissue. The lateral resolution is expected to be close to $10 \mu \mathrm{m}$.

We achieve four different incident polarization states, $H, V, P$, and $R$, by rotating the half-wave plate and the quarter-wave plate in the source arm. For each of these four incident polarization states the variable-wave plate at the reference arm is adjusted to achieve sequentially the $H, V, P$, and $R$ polarization states. The light intensities of both the source arm and the reference are measured for each of the 16

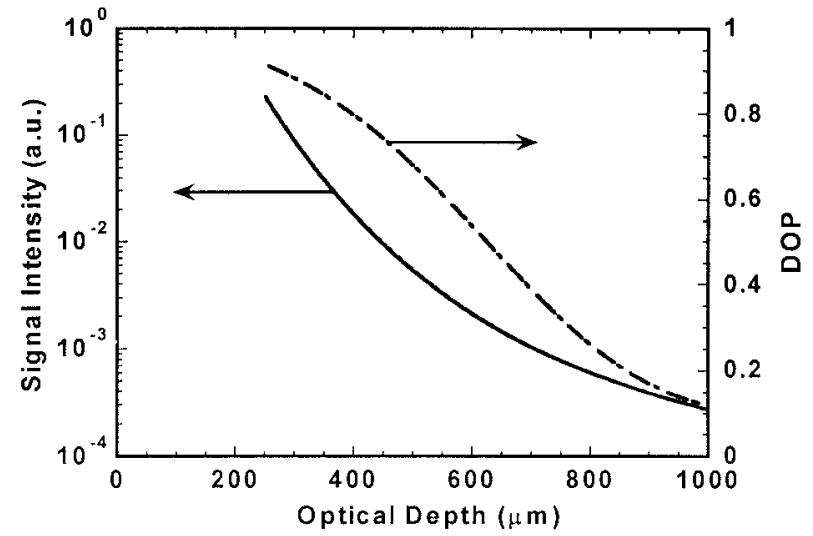

(a)

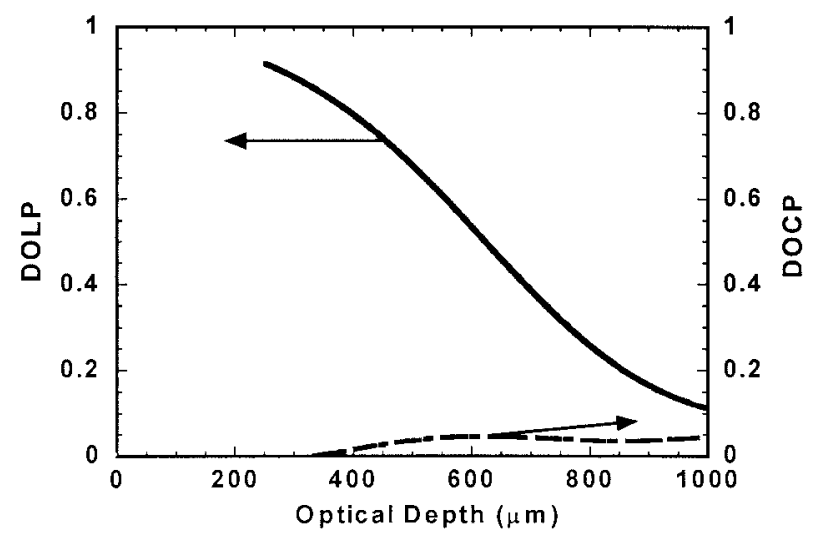

(b)

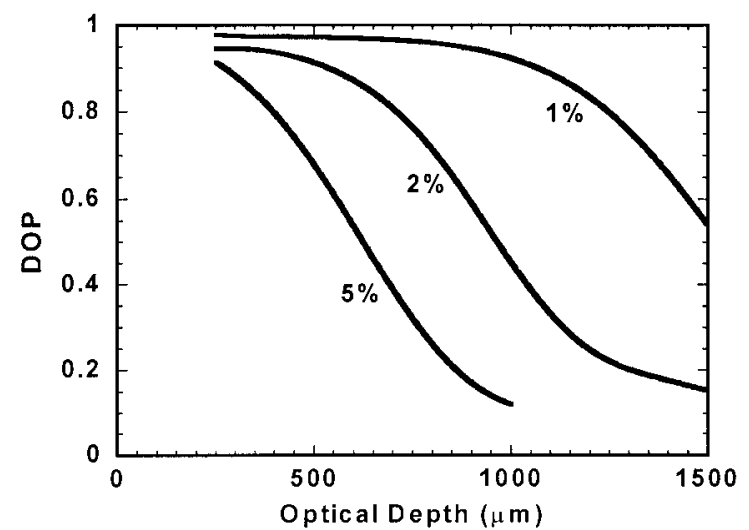

(c)

Fig. 2. Plots of (a) the horizontal linear polarization Stokes vector $S_{H 0}$ and the DOP for a $5 \%$ Intralipid solution from which the DOLP and the DOCP for (b) a 5\% Intralipid solution were calculated. (c) The DOP's for a $1 \%$, a $2 \%$, and a $5 \%$ Intralipid solution.

combinations of the polarization states in the source and the reference arms. The source intensity is measured for calibration purposes. The reference intensities are used to convert the OCT signals for calculations of the Stokes vectors and the Mueller matrices. A total of 16 polarization-sensitive OCT images are acquired and processed to obtain the 16 Mueller matrix images $M_{i j}$, in accord with Eq. (7). Alternatively, if the Stokes vector of the backscattered light is sought for a given incident polarization 


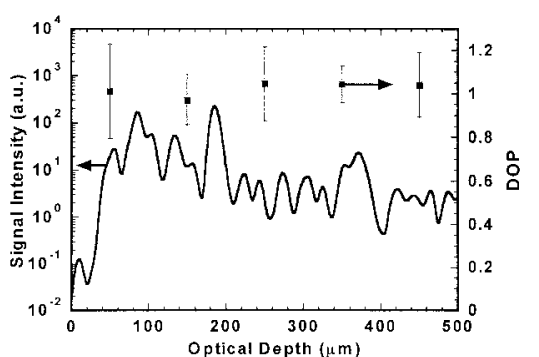

(a)

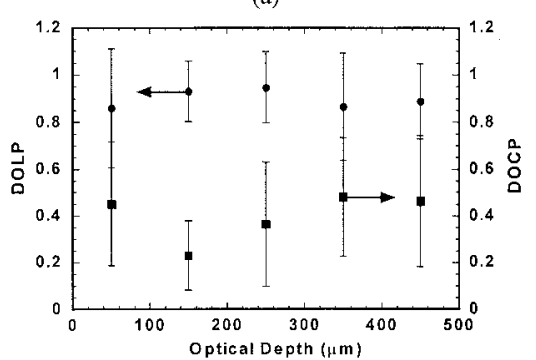

(b)

Fig. 3. Plots of (a) the horizontal linear polarization Stokes vector $S_{\mathrm{Ho}}$ and the DOP for bone tissue from the head of a yellow croaker fish and (b) the corresponding DOLP and DOCP for the same sample region.

state only four measurements need to be acquired by variation of the reference polarization state. The Stokes vector is then calculated based on Eq. (2).

The OCT system was carefully calibrated and validated. The four incident polarization states, as well as the four reference polarization states associated with each incident polarization state, were examined in terms of polarization purity. The polarization purity is defined as $I_{\min } / I_{\max }$, where $I_{\max }$ is the signal intensity of the designed polarization state and $I_{\min }$ is the intensity of the orthogonal polarization state. The measured $I_{\min } / I_{\max }$ is less than $0.15 \%$ for all the polarization states. Because the beam splitter is not an ideal polarization-independent optical element, the Mueller matrix of the beam splitter was measured for calibration. The Mueller matrices of simple optical elements measured with our setup agree with their known ideal matrices to within an error of $5 \%-10 \%$ after calibration with the measured Mueller matrix of the beam splitter.

\section{Results and Discussion}

We measured the horizontal linear Stokes vectors $\mathbf{S}_{H}$ of $1 \%, 2 \%$, and $5 \%$ Intralipid solutions and of a piece of bone tissue from the head of a yellow croaker fish with the incident light in a state of horizontal linear polarization $(H)$. The Stokes vectors were then used to calculate the DOP, the DOLP, and the DOCP for each sample. The results are shown in Figs. 2 and 3 for which the optical depth means the product between the physical depth and the refractive index of the sample. Figure 2(a) shows the $S_{H 0}$ and the DOP for the 5\% Intralipid solution; Fig. 2(b) shows the DOLP and the DOCP for the 5\% Intralipid solution; Fig. 2(c) shows the DOP for the $1 \%$, the $2 \%$, and the $5 \%$ Intralipid solutions. Figure 3(a) shows the $S_{H 0}$ and the DOP for the bone sample; Fig. 3(b) is a plot of the corresponding DOLP and DOCP. In Fig. 3 the DOP, the DOLP, and the DOCP were averaged over 100- $\mu \mathrm{m}$ segments of depth to reduce fluctuation.

All the data were averaged over 20 scans. Polynomial fitting was applied to the data for the Intralipid solutions to reduce fluctuations before calculating the Stokes vectors. The difference in the DOP is striking between the liquid and the solid samples. For the liquid samples the DOP and the DOLP decrease as the optical depth increases, as shown in Fig. 2. The DOCP has a small value that is most likely caused by noise, indicating negligible anisotropy or birefringence. The DOP decreases with an increasing concentration of Intralipid and decreases with the optical depth faster for higher concentrations of Intralipid, as shown in Fig. 2(c).

For the solid sample, however, the DOP is approximately unity throughout the detectable range of optical depths apart from the fluctuation, as shown in Fig. 3(a). The DOLP and the DOCP complement each other, as shown in Fig. 3(b), confirming the existence of optical birefringence in the sample.

The salient difference in the DOP between the liquid and the solid samples indicates that a liquid medium acts differently on our OCT signals than does a solid medium. The variation of the DOP with the optical depth and the solution concentration signifies that the apparent depolarization effect in liquid increases with the depth and the concentration. In principle, a DOP of less than unity means that the detected backscattered light is partially depolarized. Because of scattering, the completely polarized incident light is converted into nonuniformly polarized scattered light. If conventional intensity-based measurements were employed to detect the polarization properties of this nonuniformly polarized light the DOP would be less than unity and would decrease with increasing scattering. The reduction of the DOP occurs because the light that impinges upon different locations on the analyzers that are in front of the detector has different polarization states and adds in intensity after passing through the analyzers. The intensity signals of the light from the different locations, as measured with orthogonal analyzers, will partially offset each other in the calculation of the Stokes vector, whereas the total intensity measured without analyzers is always the sum of the light from all the locations.

However, OCT is an amplitude-based detection system by interference heterodyne. OCT detects the electric field of only the coherent part of the backscattered light. The electric field of the light from different locations on the detector is projected onto the analyzing polarization state and then added in amplitude. Equivalently, the electric field vectors of the light from different locations of the detector are summed, and the vector sum is then projected onto the analyzing polarization state. Because of this coherent-detection scheme in OCT, a DOP of unity is maintained despite scattering, as can be observed from the solid sample. Therefore the conventional 


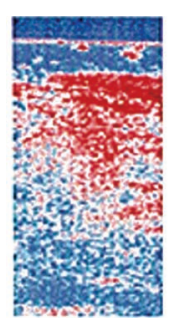

$\mathrm{RH}$

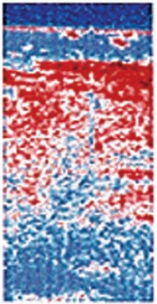

$\mathrm{HH}$
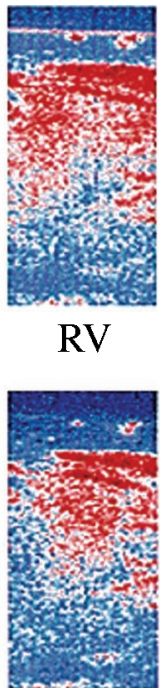

HV

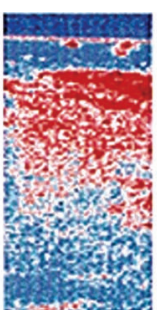

RP

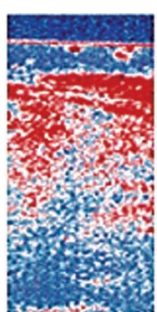

RR

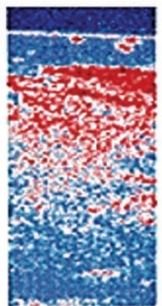

HP

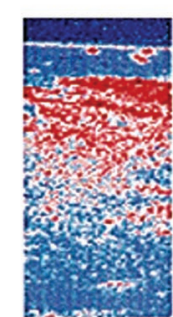

HR

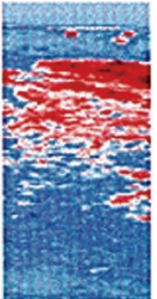

$S_{R 0}$

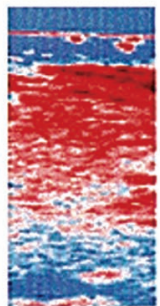

$S_{H 0}$

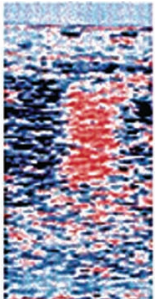

$S_{R 1}$

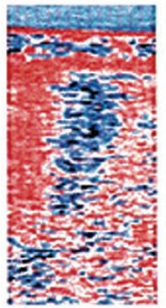

$S_{H 1}$

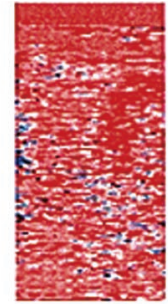

$S_{R 2}$

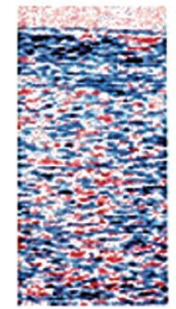

$S_{H 2}$

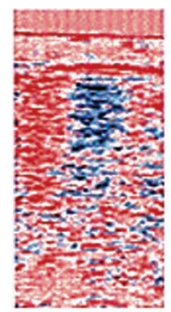

$S_{R 3}$

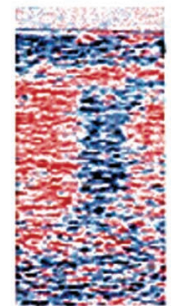

$S_{H 3}$

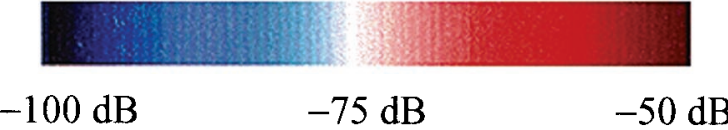

Intensity

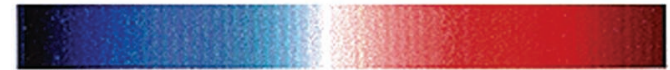

$-1$

0

$+1$

(a)

Relative Intensity

(b)

Fig. 4. (a) Raw 2-D OCT images and (b) 2-D images of the corresponding Stokes parameters of the fish-bone sample used for Fig. $3 . \quad$ All the images share the same color map. The upper boundary in each image represents the incident surface of the glass plate that was used for fixing the bone sample. The physical size of each image was $1.0 \mathrm{~mm} \times 0.5 \mathrm{~mm}$.

depolarization process in intensity-based measurements does not account for the decrease in DOP of the liquid media.

We conjecture that the decrease of the apparent DOP in liquid is caused by the Brownian motion of the scattering particles and the signal averaging during data acquisition. Brownian motion causes the polarization state of the backscattered light to fluctuate about an average state. Because our OCT system converts the interference fringes into an envelope of rectified fringes, only this positive envelope is detected and averaged.

To illustrate this point, we let $I_{H}, I_{V}, I_{P}$, and $I_{R}$ denote the intensities of the analyzed average polarization state as horizontal linear polarization, vertical linear polarization, $+45^{\circ}$ linear polarization, and right-circular polarization, respectively. Let $I_{n}$ denote the average intensity caused by the Brownian fluctuation, which is assumed, for simplicity, to be the same for all four of the measurements obtained with different analyzers. The measured Stokes vector can be expressed as

$$
\mathbf{S}^{\prime}=\left(\begin{array}{c}
I_{H}+I_{V} \\
I_{H}-I_{V} \\
2 I_{P}-\left(I_{H}+I_{V}\right) \\
2 I_{R}-\left(I_{H}+I_{V}\right)
\end{array}\right)+\left(\begin{array}{c}
2 I_{n} \\
0 \\
0 \\
0
\end{array}\right)=\mathbf{S}+\mathbf{S}_{n}
$$

The DOP of $\mathbf{S}$ is unity, whereas the DOP of $\mathbf{S}_{n}$ is zero. The DOP of $\mathbf{S}^{\prime}$ is

$$
\operatorname{DOP}\left(\mathbf{S}^{\prime}\right)=\operatorname{DOP}(\mathbf{S}) \frac{S_{0}}{S_{0}+2 I_{n}}=\frac{S_{0}}{S_{0}+2 I_{n}} .
$$

As can be seen from Eq. (11), the apparent DOP of the measured Stokes vector is less than unity. The increase in the Intralipid concentration leads to an increase in the random scattering that the light undergoes per unit optical depth. An increase in the optical depth means that the backscattered light undergoes more scattering events. The increased scattering events would cause more fluctuation because each scattering event has Brownian motion. Therefore the average intensity $I_{n}$ would increase with both the optical depth and the scatterer concentration in liquid, which would accordingly decrease the apparent DOP. This supposition can ultimately be tested if our setup is improved such that the Stokes vector of a liquid sample can be measured in a sufficiently short time period.

Two-dimensional images of the Stokes parameters for incident light of right-circular and linear horizontal polarization states are shown in Fig. 4. The sample was the piece of fish bone used for Fig. 3. The image size is $1.5 \mathrm{~mm}$ (optical depth) $\times 0.5 \mathrm{~mm}$ (lateral 


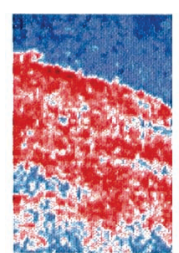

$\mathrm{HH}$

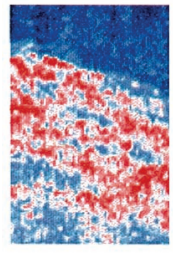

VH

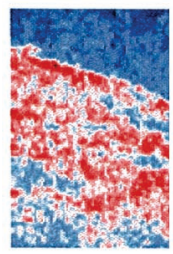

$\mathrm{PH}$

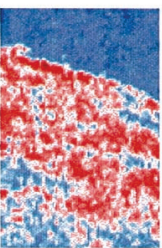

$\mathrm{RH}$

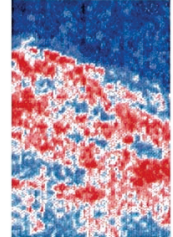

HV

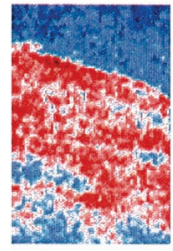

VV

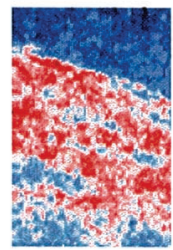

PV

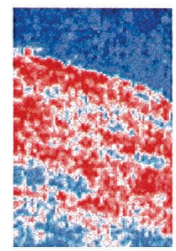

RV

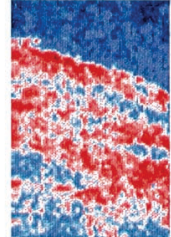

HP

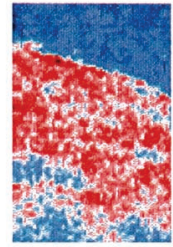

VP

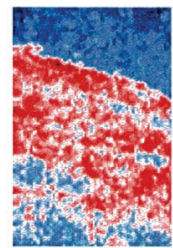

PP

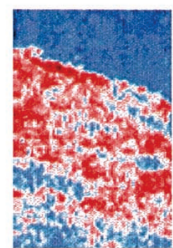

RP

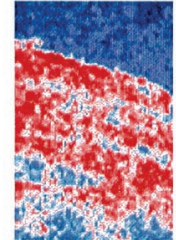

HR

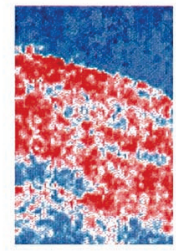

VR

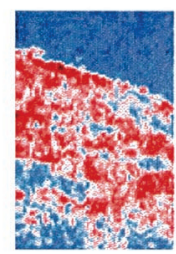

PR

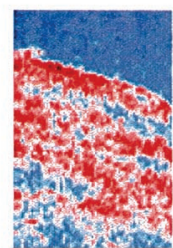

RR
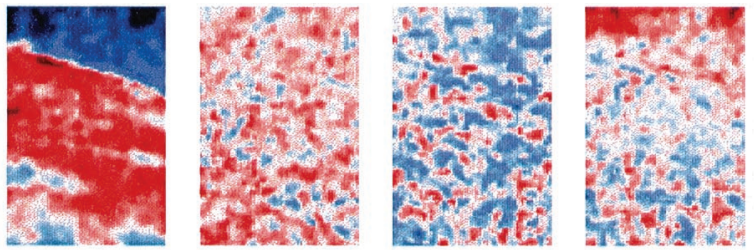

$M_{00}$

$M_{01} / M_{00}$

$M_{02} / M_{00}$

$M_{03} / M_{00}$
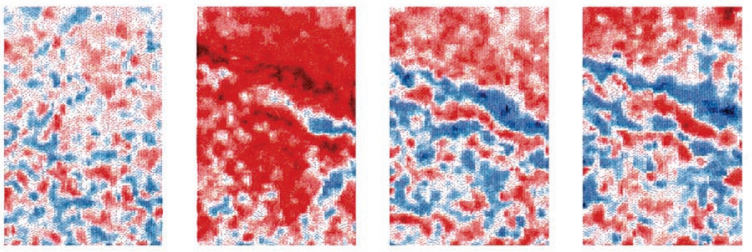

$$
M_{10} / M_{00}
$$

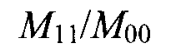

$M_{12} / M_{00}$

$M_{13} / M_{00}$
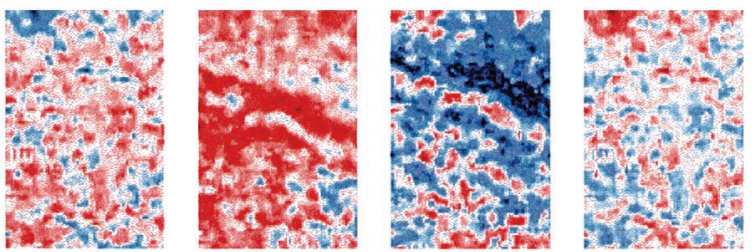

$$
M_{20} / M_{00}
$$

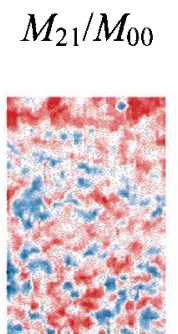

$M_{22} / M_{00}$

$M_{23} / M_{00}$
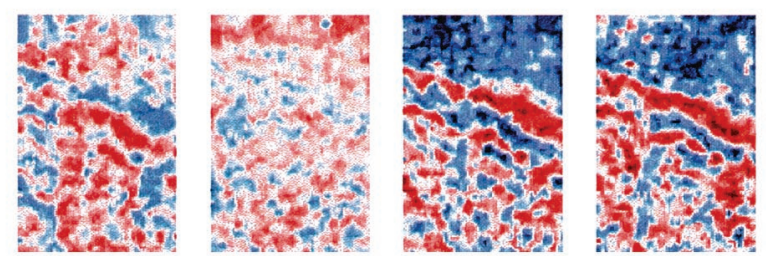

$$
M_{30} / M_{00}
$$

$M_{31} / M_{00}$

$M_{32} / M_{00}$

$M_{33} / M_{00}$

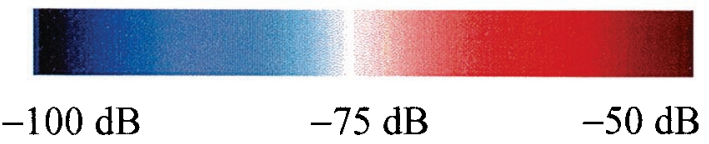

Intensity

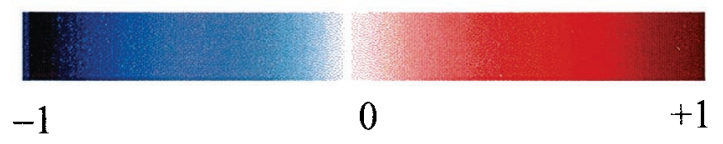

Relative Intensity

(b)

Fig. 5. (a) Raw 2-D OCT images and (b) 2-D images of the corresponding Mueller matrix parameters of the fish-bone sample used for Fig. 3. All the images share the same color map. The physical size of each image is $0.67 \mathrm{~mm} \times 0.5 \mathrm{~mm}$.

dimension). The 1.5-mm optical depth may be converted to an approximately $1.0-\mathrm{mm}$ physical depth, assuming the index of refraction of the bone sample is 1.5. The labels that represent double-polarization states in Fig. 4(a) identify OCT measurements: the source polarization state is denoted by the left-hand, letter and the reference polarization state by the right-hand letter. For example, HV refers to an OCT measurement acquired with an $H$-polarized incident field and a $V$-polarized reference field. The original 2-D image data were averaged over 20 mea- surements. From Fig. 4, we can clearly see the differences among the different elements of the Stokes vector. The four $S_{1}$ and $S_{3}$ images reveal some structures in the central region of approximately 0.2 $\mathrm{mm} \times 0.5 \mathrm{~mm}$ that are not seen in the other four Stokes images. The structures in the four $S_{1}$ and $S_{3}$ images of both incident polarization states are similar in both shape and size. However, the central region in $S_{R 1}$ has a higher intensity than does the surrounding region and looks solid, whereas the central regions in $S_{R 3}, S_{H 1}$, and $S_{H 3}$ have lower intensi- 
ties than do the surrounding regions and look like voids. The central imaged region of the sample must have optical polarization properties different from those of the surrounding region, and it must change the polarization state of the backscattered light from this region in a dissimilar manner. The change in polarization state can be attributed to both optical birefringence and scattering.

We measured the 2-D Mueller matrix images of another region of the bone sample. The raw images and the images of the corresponding 16 Mueller matrix elements are shown in Fig. 5. A total of 16 measurements were acquired. The four Stokes vectors corresponding to the four incident polarization states were first calculated by use of Eq. (2) and then were used to calculate the Mueller matrix, according to Eq. (7). The Mueller matrix images were compensated with the Mueller matrix of a sample mirror and the beam splitter to account for the polarization effects of the beam splitter. The compensation Mueller matrix was measured as

$$
\mathbf{M}_{\text {cal }}=\left[\begin{array}{crrr}
1.000 & -0.0420 & -0.0028 & -0.0479 \\
-0.0405 & 0.9975 & -0.0591 & -0.0306 \\
-0.0095 & -0.0004 & 1.0671 & 0.2089 \\
-0.0134 & -0.0182 & 0.2008 & 1.0999
\end{array}\right] .
$$

The image size is $1.0 \mathrm{~mm}$ (optical depth) $\times 0.5 \mathrm{~mm}$ (lateral dimension). The 1.0-mm optical depth can be converted to an approximately $0.67-\mathrm{mm}$ physical depth, assuming the index of refraction of the bone sample is 1.5. From the 16 raw OCT images [Fig. 5(a)] the degradation effects on the incident polarization state can clearly be seen. Some regions of the $\mathrm{HV}$ and the $\mathrm{VH}$ images have strong cross-polarized signals, which appear as red spots in the images, and the corresponding locations in the copolarized $\mathrm{HH}$ and VV images have strong signals as well. Therefore the incident light is partially converted to the cross-polarization state by the clusters in these regions. In most other regions the backscattered light still preserves most of the original polarization state because the copolarized signals are much stronger than the cross-polarized signals.

Processed $4 \times 4$ Mueller matrix images are shown in Fig. 5(b). The image of the Mueller matrix element $M_{00}$ corresponds to a polarization-independent image, as acquired by a nonpolarization OCT system. The other Mueller matrix elements $M_{i j}$ are pixelwise normalized by the $M_{00}$ image. The polarizationindependent element $M_{00}$ reveals significantly less information than do the other elements, as can clearly be seen from Fig. 5(b). Strong layered structures are clearly seen in some of the images, such as $M_{12}, M_{13}, M_{22}, M_{32}$, and $M_{33}$.

Only the Mueller matrices of solid samples were measured to determine stability considerations because soft samples would vibrate within the current acquisition time. After the scanning speed is increased and the system is fully automated, we should be able to measure soft-tissue samples, which would greatly enhance the application of this technique. The technique can furnish depth-resolved Mueller matrix characterizations of native biological tissues either in vivo or in vitro with high spatial resolution. Analysis of the Mueller matrix can extract information on the origin of the polarization effect, which is related to the local anisotropic structure within the sample. Detailed interpretations of the Mueller matrices can reveal much more information than can conventional OCT about the local structures. Further investigations are warranted in this direction.

\section{Conclusion}

In summary, we have demonstrated a novel imaging technique that measures the Stokes vectors and the Mueller matrices with OCT in biological tissues or other turbid media. With this technique, we have measured the DOP, the DOLP, and the DOCP of liquid and solid samples. The DOP maintains a value of unity within the detectable depth for solid samples, whereas the DOP decreases with the optical depth for the liquid sample. This difference in the behavior of liquids is probably related to the Brownian motion of the scattering particles in liquids. The Brownian motion of the particles causes random variations of the polarization state of the incident light. The 2-D images of the Stokes vectors and the Mueller matrices have revealed more information about the tissue samples than has standard OCT. The Mueller matrix imaging system has potential applications in both scientific research and clinical practice.

This study was sponsored in part by National Institutes of Health grants R29 CA68562, R01 CA71980, and R21 CA83760; by National Science Foundation grant BES-9734491; and by Texas Higher Education Coordinating Board grant 000512-0123-1999.

\section{References}

1. W. Drexler, U. Morgner, F. X. Kärtner, C. Pitris, S. A. Boppart, X. D. Li, E. P. Ippen, and J. G. Fujimoto, "In vivo ultrahighresolution optical coherence tomography," Opt. Lett. 24, 12211223 (1999).

2. A. Rollins, S. Yazdanfar, M. Kulkarni, R. Ungarunyawee, and J. Izatt, "In vivo video rate optical coherence tomography," Opt. Express 3, 219-229 (1998), http://epubs.osa.org/opticsexpress.

3. M. J. Everett, K. Schoenenberger, B. W. Colston, Jr., and L. B. Da Silva, "Birefringence characterization of biological tissue by use of optical coherence tomography," Opt. Lett. 23, 228-230 (1998).

4. J. F. de Boer, S. M. Srinivas, A. Malekafzali, Z. Chen, and J. S. Nelson, "Imaging thermally damaged tissue by polarizationsensitive optical coherence tomography," Opt. Express 3, 212218 (1998), http://epubs.osa.org/opticsexpress.

5. J. M. Schmitt and S. H. Xiang, "Cross-polarized backscatter in optical coherence tomography of biological tissue," Opt. Lett. 23, 1060-1062 (1998).

6. J. F. de Boer, T. E. Milner, and J. S. Nelson, "Determination of the depth-resolved Stokes parameters of light backscattered from turbid media by use of polarization-sensitive optical coherence tomography," Opt. Lett. 24, 300-302 (1999).

7. G. Yao and L. V. Wang, "Two-dimensional depth-resolved Mueller matrix characterization of biological tissue by optical coherence tomography," Opt. Lett. 24, 537-539 (1999).

8. W. S. Bickel and W. M. Bailey, "Stokes vectors, Mueller matrices, and polarized scattered light," Am. J. Phys. 53, 468-478 (1995). 